\title{
Manipulating Bloch surface waves in 2D: a platform concept-based flat lens
}

\author{
Libo Yu, Elsie Barakat, Tristan Sfez, Lubos Hvozdara, Joab Di Francesco and Hans Peter Herzig
}

At the end of the 1970s, it was confirmed that dielectric multilayers can sustain Bloch surface waves (BSWs). However, BSWs were not widely studied until more recently. Taking advantage of their high-quality factor, sensing applications have focused on BSWs. Thus far, no work has been performed to manipulate and control the natural surface propagations in terms of defined functions with two-dimensional (2D) components, targeting the realization of a 2D system. In this study, we demonstrate that 2D photonic components can be implemented by coating an in-plane shaped ultrathin $(\sim \lambda / 15)$ polymer layer on the dielectric multilayer. The presence of the polymer modifies the local effective refractive index, enabling direct manipulation of the BSW. By locally shaping the geometries of the 2D components, the BSW can be deflected, diffracted, focused and coupled with 2D freedom. Enabling BSW manipulation in 2D, the dielectric multilayer can play a new role as a robust platform for $2 \mathrm{D}$ optics, which can pave the way for integration in photonic chips. Multiheterodyne near-field measurements are used to study light propagation through micro- and nano-optical components. We demonstrate that a lens-shaped polymer layer can be considered as a 2D component based on the agreement between near-field measurements and theoretical calculations. Both the focal shift and the resolution of a 2D BSW lens are measured for the first time. The proposed platform enables the design of 2D all-optical integrated systems, which have numerous potential applications, including molecular sensing and photonic circuits.

Light: Science \& Applications (2014) 3, e124; doi:10.1038/Isa.2014.5; published online 3 January 2014

Keywords: Bloch surface wave; 2D optics; manipulation; micro- and nano-optics; nanophotonics; platform

\section{INTRODUCTION}

One or several elements are considered to comprise a two-dimensional (2D) optical system if they fulfill two conditions. First, the in-plane light propagation must have two spatial non-imaginary propagation constants. Second, the corresponding optical elements should have a $2 \mathrm{D}$ degree of freedom in shape. The previous statements may appear to imply that the reduction from three-dimensional (3D) to $2 \mathrm{D}$ is primarily a reduction in the degree of freedom. However, one of the main advantages is that $2 \mathrm{D}$ elements can have arbitrary shapes, which is difficult to achieve in 3D.

There are various methods for addressing a $2 \mathrm{D}$ optical environment. One approach is represented by the use of wave-guiding media wherein the light is confined and propagated in a sandwiched structure. However, in the case of slab waveguides, the light is almost completely buried in the inner layers of the waveguide; thus, direct spatial mapping remains difficult or impossible. As an alternative to waveguides, a second route for $2 \mathrm{D}$ optics is represented by surface plasmons (SPPs) on smooth planar or structured metallic films. SPPs are electronic-electromagnetic modes sustained at an appropriate metallic/dielectric interface wherein the field reaches its maximum intensity at the surface of the metal. ${ }^{1-3}$ Extensive studies have reported on 2D optical elements based on SPPs, including SPP scattering, ${ }^{4-6}$ Bragg mirrors, ${ }^{7,8}$ lenses, ${ }^{9-13}$ interferometers and resonators. ${ }^{14,15}$ However, 2D optics based on SPPs are intrinsically limited by absorption losses due to metals, which reduce the propagation length of SPPs.

In contrast, Bloch surface waves (BSWs) ${ }^{16}$ can be considered as the dielectric analogue of SPPs with less loss. BSWs are electromagnetic surface waves excited at the interface between a truncated periodic dielectric multilayer and a surrounding medium. SPP-like sensors based on BSWs are considered as an alternative to SPPs, ${ }^{17}$ and graphene-based one-dimensional photonic crystals are considered as an alternative to graphene SPPs. ${ }^{18}$ Furthermore, BSWs may potentially be applied to surface-enhanced Raman spectroscopy. ${ }^{19,20}$ Based on previous works, we can deduce that BSWs may be a good candidate for $2 \mathrm{D}$ optical integration, similar to SPPs. Previous works on BSWs have primarily focused on far-field sensing applications. Extensive studies and applications of BSWs only recently began receiving attention within a few research groups, 20 years after BSWs were first examined at the end of the $1970 \mathrm{~s}^{21}$ Studies of the specific BSW sensing properties have been proposed and demonstrated, including an experimental determination of BSW sensitivity, ${ }^{22}$ a direct comparison of BSW and SPP sensors ${ }^{23}$ and the experimentally achieved improvement of sensitivity with BSWs in comparison to SPPs. ${ }^{24}$ Sensing applications include chemical and biosensing, ${ }^{25-27}$ gas sensing ${ }^{28}$ and fluorescence emission enhancement. ${ }^{29-31}$ 
Although a dielectric multilayer is interesting for surface sensing applications, it has never been considered for 2D integrated optics. To date, no work has been done to manipulate and control BSW propagation in terms of $2 \mathrm{D}$ photonic devices to realize $2 \mathrm{D}$ systems with well-defined optical functionalities. The concept of the multilayer as a fundamental platform for 2D integrated optics providing sensing functionalities has received attention in recent works performed at the OPT Laboratory by means of a multiheterodyne scanning nearfield optical microscope (SNOM) ${ }^{32,33}$ The propagation of BSWs on a planar multilayer, ${ }^{34}$ the lateral confinement of BSWs on ultrathin polymeric ridges, ${ }^{35}$ the near-field mapping of standing BSWs on shallow Bragg gratings on a multilayer ${ }^{36}$ and BSW refraction through waveguide structures ${ }^{37}$ are just few of the subjects recently addressed on this topic.

In this study, we propose and demonstrate that the dielectric multilayer can be endowed with a new role as a universal platform concept, wherein the manipulation of BSWs can be achieved and various 2D photonic components can be reproduced on the platform with ease. The primary benefit of this approach is that these flat photonic components can have arbitrary shapes, which are generally more difficult to fabricate and control in 3D. A further benefit of the platform concept is that thin film multilayers enable standard wafer-scale production. The top surface can be modified to customize a $2 \mathrm{D}$ micro-system using e-beam writing, optical lithography, stamping or other replication techniques. Furthermore, we discuss the intrinsic differences between $2 \mathrm{D}$ and $3 \mathrm{D}$ optical phenomena.

There are several advantages provided by BSW propagation on nearly flat surfaces. First, due to the use of dielectric material, the losses are very low, thus allowing for the propagation of BSWs over long distances. Another advantage in using BSWs is the possibility of operating within a broad range of wavelengths, by properly designing a suitable multilayered structure. Furthermore, because the maximum intensity associated with the BSW can be tuned on the surface, a strong field intensity, increased by several orders of magnitudes, can be achieved and can thereby enhance the field close to the structure surface. This tunable localized field confinement is particularly attractive for fluorescent biosensing.

In our work, we demonstrate that $2 \mathrm{D}$ photonic components can be implemented by coating an in-plane shaped ultrathin $(\sim \lambda / 15)$ polymer layer on a multilayer. The presence of the polymer modifies the local effective refractive index, enabling a direct manipulation of the BSW. By locally shaping the geometries of the 2D photonic components, the BSW can be diffracted, focused, coupled and resonated with $2 \mathrm{D}$ freedom. A multiheterodyne $\mathrm{SNOM}^{32,33}$ developed in our lab (Optics \& Photonics Technology Laboratory, Ecole Polytechnique Fédérale de Lausanne) is used to monitor the near-field behavior on the examined platform and a lens-shaped polymer layer. The property of the platform defines the propagation of the surface modes and has a significant impact on the capability of manipulation. We demonstrate that the lens-shaped polymer layer can be considered as a complete 2D component based on the agreement between near-field measurements and theoretical calculations. Both the focal shift and the resolution of a BSW 2D lens are measured for the first time. This manuscript is organized as follows: the next section describes the device and the experimental framework. The propagation properties of the surface modes defined by the platform and their effect on the manipulation capability are studied. As a demonstration of this platform, a 2D BSW lens is investigated in the subsequent section. Finally, the conclusions summarize and highlight the optimization of key parameters.

\section{MATERIALS AND METHODS}

\section{Device, experimental setup and BSW properties}

BSW confinement is strongly dependent on the polarization. However, the device can be designed for both transverse electric (TE) and transverse magnetic (TM) polarization. In this work, the multilayer is meant to work in TE polarization, at a center wavelength of $1500 \mathrm{~nm}$. The center wavelength is chosen by adjusting the incident angle in order to excite the BSW at the wavelength range of the laser used in our experiments $(1460-1580 \mathrm{~nm})$. The platform is based on a 1D photonic crystal, composed of dielectric stacks with alternating refractive indices. The platform consists of six pairs of silicon dioxide and silicon nitride, with refractive indices of 1.45 and 1.79 at $\lambda=1500 \mathrm{~nm}$, respectively, deposited on a glass wafer as a Bragg mirror (Figure 1a). The thicknesses of the layers are $492 \mathrm{~nm}$ and $263 \mathrm{~nm}$, respectively. An 80-nm-thick layer of silicon nitride is also deposited on top of the Bragg mirror, which will hereafter be called the top layer. A 100-nm-thick positive photoresist (PR) (AZ1518, $n=1.458)$ layer is deposited on the platform (Bragg mirror + top layer) and is used to shape the surface components. We choose AZ1518 in order to develop different integrated optical functions with complex shaped photonic components for the future circuit industry. Furthermore, the PR can be easily fabricated and deposited, which allows us to obtain reproducible devices. The designed structures of the $2 \mathrm{D}$ photonic components are patterned into the PR layer by UV photolithography.

The BSW must be coupled under a condition of total internal reflection, usually with a Kretchmann configuration using a BK7-glass prism $\left(n_{\mathrm{g}}=1.501\right)$, in order to match the propagation constant of the BSW, as shown in Figure 1a. The incident beam, at an angle $\theta$ with respect to the normal of the platform, should match the condition of $\beta=k \sin \theta$ to allow for excitation and propagation of the BSW in the $y-z$ plane. $k=n_{g} \times 2 \pi / \lambda$ is the wave vector of the incident beam, $\beta$ is the wave vector of the BSW and $\theta$ is the incident angle. For a chosen incident angle of $\theta=52.2^{\circ}$, the corresponding wavelength is $1542.2 \mathrm{~nm}$. The incident beam is injected from the glass side of the platform. The projection of the focused spot on the platform has a diffraction-limited spot diameter of $30 \mu \mathrm{m}$ in the coupling zone and propagates in the $z$ direction. The photonic components made by the PR are located several hundred microns from the center of the coupling zone ( $h$ in Figure 1a). The field information of the light distribution is recorded using a SNOM in collection mode with an aperture probe attached to a single-mode optical fiber. A CCD camera is used to detect the reflected light in the far-field.

The near-field intensities for the TE and TM polarizations can be directly compared with the aid of the MH-SNOM. Figure $1 \mathrm{~b}$ shows the experimentally normalized amplitude of the evanescent field measured at the coupling zone for both TE and TM polarizations through the multilayer without a PR layer. The results show that the ratio between the maximum amplitude of the two signals is approximately 10, which is approximately fourfold greater than the values reported in Ref. 34. Note that in addition to the design of the multilayer, the experimentally observed field enhancement is strongly dependent on the coupling configuration. In our case, we use a Gaussian beam with a divergence of $4^{\circ}$ to create a coupling zone on the surface of the multilayer. The corresponding coupling efficiency is estimated to be approximately $6 \%$ according to. ${ }^{38}$ The enhancement effect can be directly observed by using a collimated beam of incidence. ${ }^{34}$ However, the observed field enhancement is not optimized. This factor can be increased by tailoring the top layer of the photonic crystal. This feature is interesting for sensing applications in which orthogonal polarization is usually applied as 


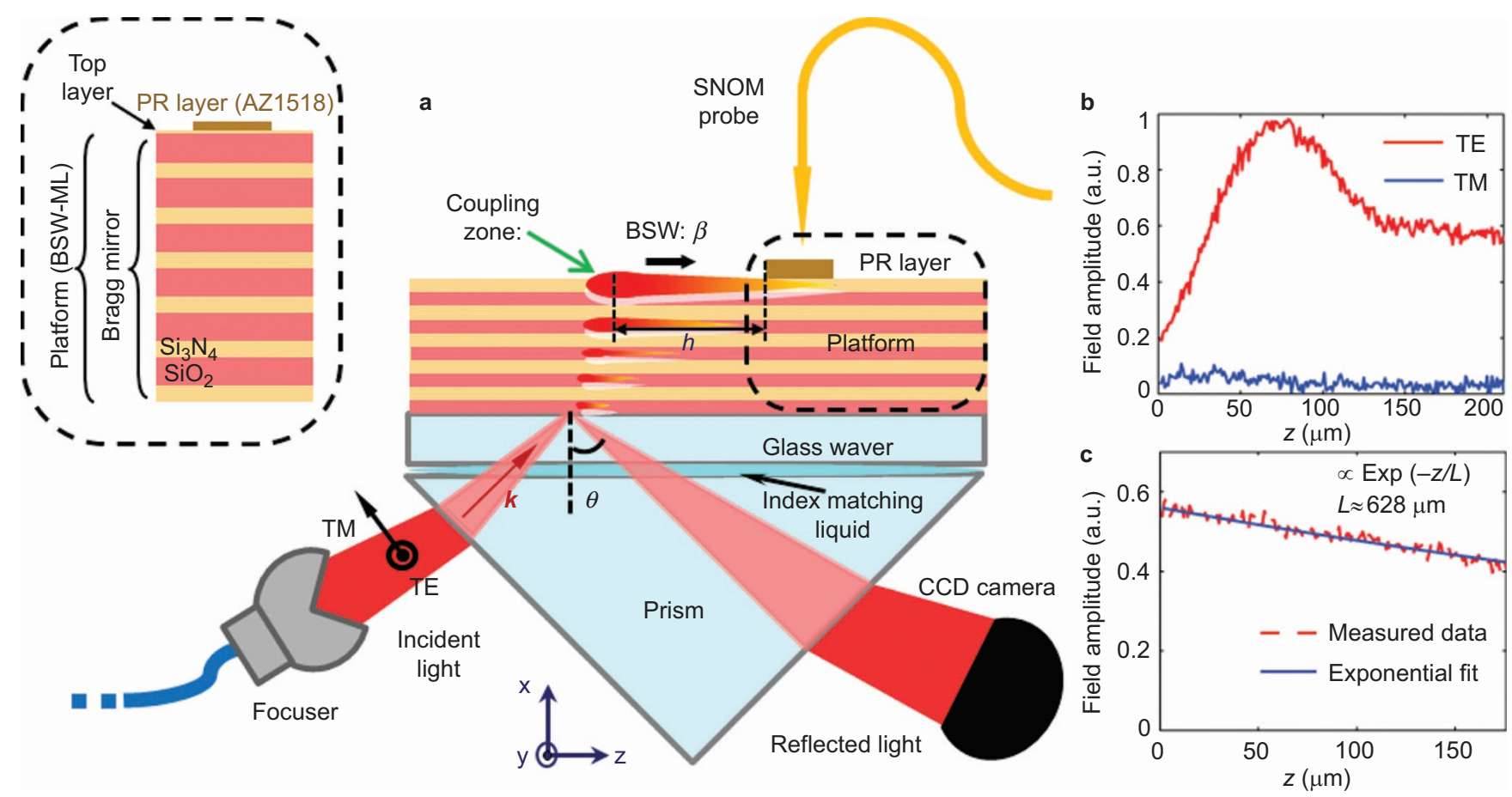

Figure 1 (a) BSW excitation is performed in the Kretschmann configuration using a BK7-glass prism. $\theta$ is the incident angle, $k$ is the wave vector of the incident beam, $\beta$ is the wave vector of the BSW and $h$ is the distance between the surface photonic components and the coupling spot. The SNOM probe collects light in the near-field on top of the 2D structures, whereas the CCD camera measures light reflected in the far-field. The inset shows that the platform consists of six layers of silicon nitride (263 nm, $n=1.79$ ) and silicon dioxide ( $492 \mathrm{~nm}, n=1.45$ ) and a top layer of silicon nitride $(80 \mathrm{~nm})$. The 2D photonic components are produced from photoresist coated on the platform. (b) Measured electric field amplitude for TE and TM polarizations at the coupling zone as a function of the propagation distance. (c) Decay length $L$ obtained by fitting the field amplitude of the propagating BSW. BSW, Bloch surface wave; 2D, two-dimensional; SNOM, scanning near-field optical microscope; TE, transverse electric; TM, transverse magnetic.

a reference. Another important property of BSWs is the decay length $L$, which indicates the attenuation distance of the BSW in the plane in the form of $\exp (-z / L)$. The experimental decay length

on the platform is $628 \mu \mathrm{m}$, as obtained by fitting the decrease of the field amplitude with distance by an exponential law, as shown in Figure 1c. This decay is caused by the absorption of the material,
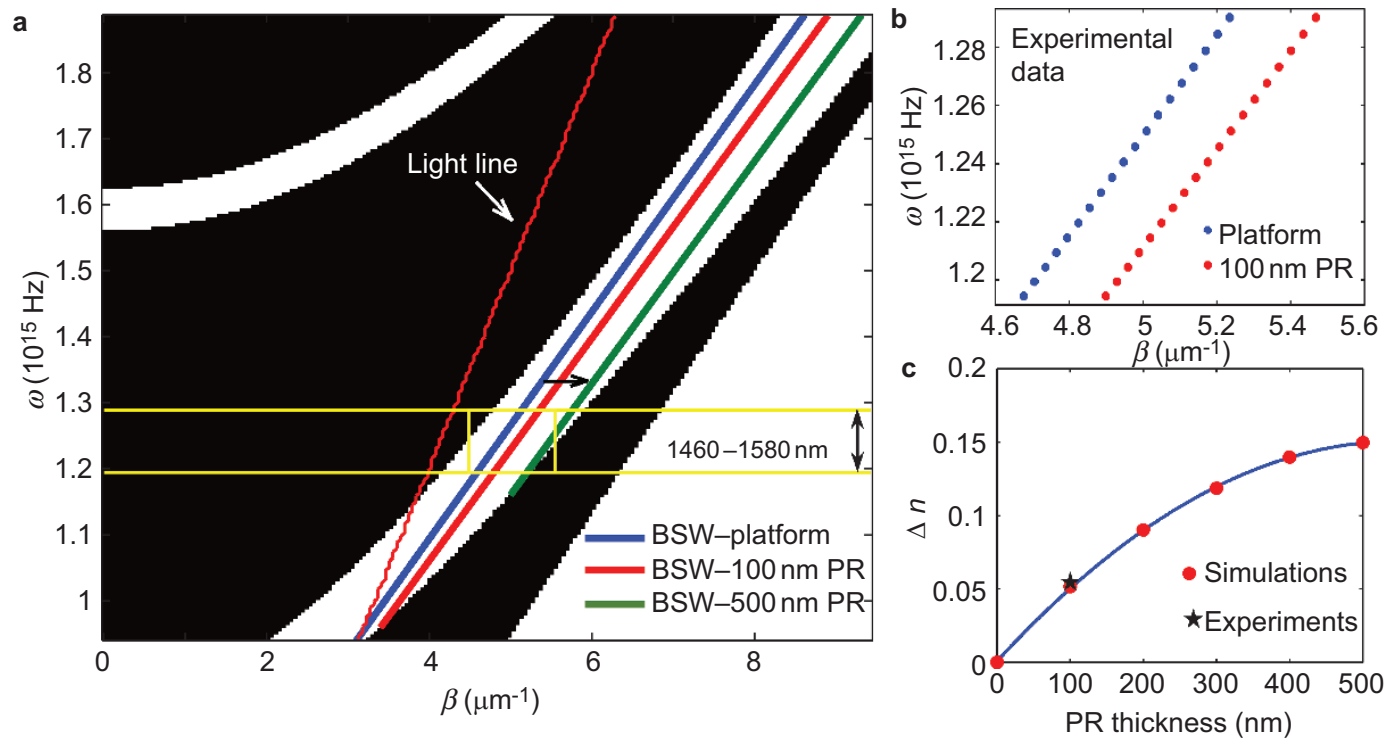

Figure 2 (a) Band gap diagram of the multilayer with semi-infinite stacks calculated for TE polarization. The white area indicates the location of the forbidden band. Light line and dispersion curves of the BSW for the platform alone and for the platform with a 100-nm/500-nm PR coating are plotted, respectively. (b) Experimental dispersion relations of the bare multilayer (platform) and the case with a 100-nm-thick PR layer coating are obtained by detecting the far-field leakage in the reflected beam for varying $\theta$ and $\lambda$. (c) The effective index contrast of the BSW between the two media, namely, the platform (PR thickness $=0)$ and the coated layers (PR thickness: 100-500 nm), are extracted from RCWA simulations. The star indicates the measured $\Delta n$ deduced from the measured dispersion curve in (b) for a 100-nm PR coating. BSW, Bloch surface wave; PR, photoresist; RCWA, rigorous coupled-wave analysis; TE, transverse electric. 
surface scattering and leakage into the prism. Silicon nitride shows low absorption in the near infrared, and because the fabrication shows low roughness of the multilayer surfaces, the surface mode is weakly scattered by the film roughness. The BSW amplitude is essentially attenuated by leakage into the $\operatorname{prism}^{38}$ due to the design of the multilayer, which allows for tunneling. This property is required for the Kretschmann configuration. Nevertheless, the measured value is seven times greater than the value recently obtained for the 'long-range SPPs' studied by Lin et al. ${ }^{39}$ confirming the low loss property of the BSW. Further loss reduction should be possible by using another design of the multilayer and choosing an alternative coupling arrangement.

\section{BSW manipulation impacted by the platform}

The property of the platform defines the propagation of the surface modes and has an essential impact on the capability of manipulation. The band diagram of the underlying multilayer with semi-infinite stacks is calculated with the matrix method ${ }^{16}$ and is illustrated in Figure 2a. The $\beta$ axis is the constant for propagation parallel to the layers, and the $\omega$ axis $(\omega=2 \pi c / \lambda)$ represents the frequency of light propagating in vacuum. The white area indicates the forbidden band. The BSW dispersion curves are calculated with the rigorous coupled-wave analysis method. The given parameters of the platform (Bragg mirror+top layer) are chosen so that the dispersion relation of the mode lies in the band gap of the Bragg mirror (below the light line), and thus, the mode can be confined at the top layer. The thickness of the top layer defines the location of the dispersion line in the band gap and, consequently, the properties of the propagation mode on the platform. The thickness of the PR layer is chosen to tailor the surface mode of the platform; hence, the thickness leads to variations in the optical properties of the 2D photonic components. The BSW dispersion line moves rightwards inside the band gap as the PR thickness increases (Figure 2a). The results show that the tunability of the propagation constant of the BSW mode in the PR layer is limited by the band gap of the underlying Bragg mirror. For a center wavelength of $1500 \mathrm{~nm}$, this limit corresponds to a maximum PR thickness of $500 \mathrm{~nm}$ in our design. In this work, a 100-nm PR layer is coated for the first attempt because this layer is thick enough to guide the BSW on the platform and thin enough to exhibit observable evanescent tails. The platform is fabricated using plasma-enhanced chemical vapor deposition.

By adding a PR layer to the platform, we introduce a refractive index contrast $\Delta n$ of the BSW between the PR layer and the platform. This refractive index contrast $\Delta n$, which can be deduced from the dispersion relations, plays a key role in controlling the propagation of the BSW on the platform. The experimental dispersion relations in Figure $2 \mathrm{~b}$ are obtained by detecting the far-field leakage in the reflected beam for varying $\theta$ and $\lambda$. Because the experiment is carried out using a mode-hop-free tunable laser designed for the C-band, the experimental dispersion relation is limited to a wavelength range of 1460 $1580 \mathrm{~nm}$. The BSW wave vector $\beta$ is defined as the product of $\omega / c$ and $n_{\mathrm{BSW}}$, where $n_{\mathrm{BSW}}$ is the refractive index of the BSW, and the effective index of the BSW on the medium can be deduced from

$$
n_{\mathrm{BSW}}=\frac{\beta \mathrm{c}}{\omega}
$$

Thus, for a constant wavelength, we can deduce the effective index of the BSW for both the uncoated and PR-coated platform $\left(n_{\mathrm{p}}\right.$ and $n_{\mathrm{PR}}$, respectively) from the measured dispersion curves. At $\lambda=1542.2 \mathrm{~nm}$, $\omega=2 \pi c / \lambda$ is fixed, and $\beta$ is obtained from the measured curve in Figure $2 \mathrm{~b}$. The refractive index of the BSW on the bare multilayer (platform) and the PR layer is then deduced from Equation (1) at $n_{\mathrm{p}}=1.1862$ and $n_{\mathrm{PR}}=1.2415$, respectively. The simulated refractive index contrast $\Delta n$ as a function of the PR thickness is extracted from rigorous coupled-wave analysis simulations, as shown in Figure 2c. The experimental refractive index contrast $\Delta n=n_{\mathrm{PR}}-n_{\mathrm{p}}=0.055$ for a 100 $\mathrm{nm}$ PR layer matches the corresponding simulated value. It can be seen that by increasing the PR thickness, the refractive index contrast $(\Delta n)$ increases and reaches 0.15 for a PR thickness of $500 \mathrm{~nm}$. Note that at the center wavelength, the PR thickness must be kept $<500 \mathrm{~nm}$ to remain within the forbidden band gap, which is a critical condition for BSW generation (Figure 2a). For the same multilayer, the top layer thickness can be designed to tune the dispersion curve position to the lower limit of the band gap. Under this condition, we can achieve a refractive index contrast, $\Delta n$, of 0.25 . To increase this value, one can enlarge the band gap energy by increasing the refractive index contrast between the different layers defining the multilayer. For example, by replacing the silicon nitride with lithium niobate $(n \approx 2.143)$, the band gap energy is enlarged, and a $\Delta n$ of 0.5 can be achieved.

\section{RESULTS AND DISCUSSION}

A significant advantage of $2 \mathrm{D}$ photonic components is that we can integrate nearly arbitrary optical structures and observe light propagation through the structures using near-field measurements. We demonstrate the potential of the platform concept by studying a planoconvex refractive lens.

\section{Two-dimensional lens}

First, a fundamental investigation of light propagation through 2D photonic components is performed in the near-field on a 2D planoconvex lens appended to a waveguide (Figure $3 \mathrm{~b}$ ). The multilayer is illuminated by an oblique incident beam at $\lambda=1542.2 \mathrm{~nm}$. The lens is then illuminated by the generated BSW at the surface of the platform. The lens is fabricated by UV lithography, with a depth and radius of $R=8.5 \mu \mathrm{m}$; the width of the waveguide is $2 R$, and its length is $L=50 \mu \mathrm{m}$. The distance between the coupling zone and the first interface of the lens is set to $h=214 \mu \mathrm{m}$ to avoid a fast decay. The planoconvex lens focuses the propagating BSW on the surface of the platform (schematic in Figure 3b). The topography of the convex lens is given in Figure $3 \mathrm{a}$.

The simulated near-field distribution shown in Figure 3d is calculated using CST Microwave Studio, based on the finite-difference time-domain method. Because the incident beam, which has a diameter of $30 \mu \mathrm{m}$ in the coupling zone, is much larger than the lens diameter $(17 \mu \mathrm{m})$, the incident beam is considered as a plane wave. A $2 \mathrm{D}$ lens geometry is constructed to simulate the behavior of the BSW, neglecting the losses due to outcoupling (leakage) of the light through the multilayer. The measured and simulated field distributions at the vicinity and on the top of the lens are displayed in Figure $3 \mathrm{c}$ and $3 \mathrm{~d}$, respectively, which show good agreement. In both cases, the intensity is normalized to the incident field in front of the lens. We call the distance between the maximum intensity position and the first interface of the lens the focal distance (FD) (see the marked 'FD' in Figure 3d). We experimentally observe a focal distance of $60 \pm 1 \mu \mathrm{m}$, which matches the simulated value of $60.7 \mu \mathrm{m}$.

These results are very different from the geometrical (paraxial) focal distance $f_{g}=181.74 \mu \mathrm{m}$ to the focal spot. Both $f_{g}$ and FD are measured from the first interface of the lens. The measured focal distance is only $33 \%$ of the geometrical focal distance because we have a small lens in terms of its Fresnel number $(N)$. Generally, lenses are characterized by the Fresnel number $N\left(N=R^{2} / \lambda f\right)$, indicating the diffraction effects of the lens at a specific wavelength $\lambda$, where $R$ is the lens radius and $f$ is the focal length. 

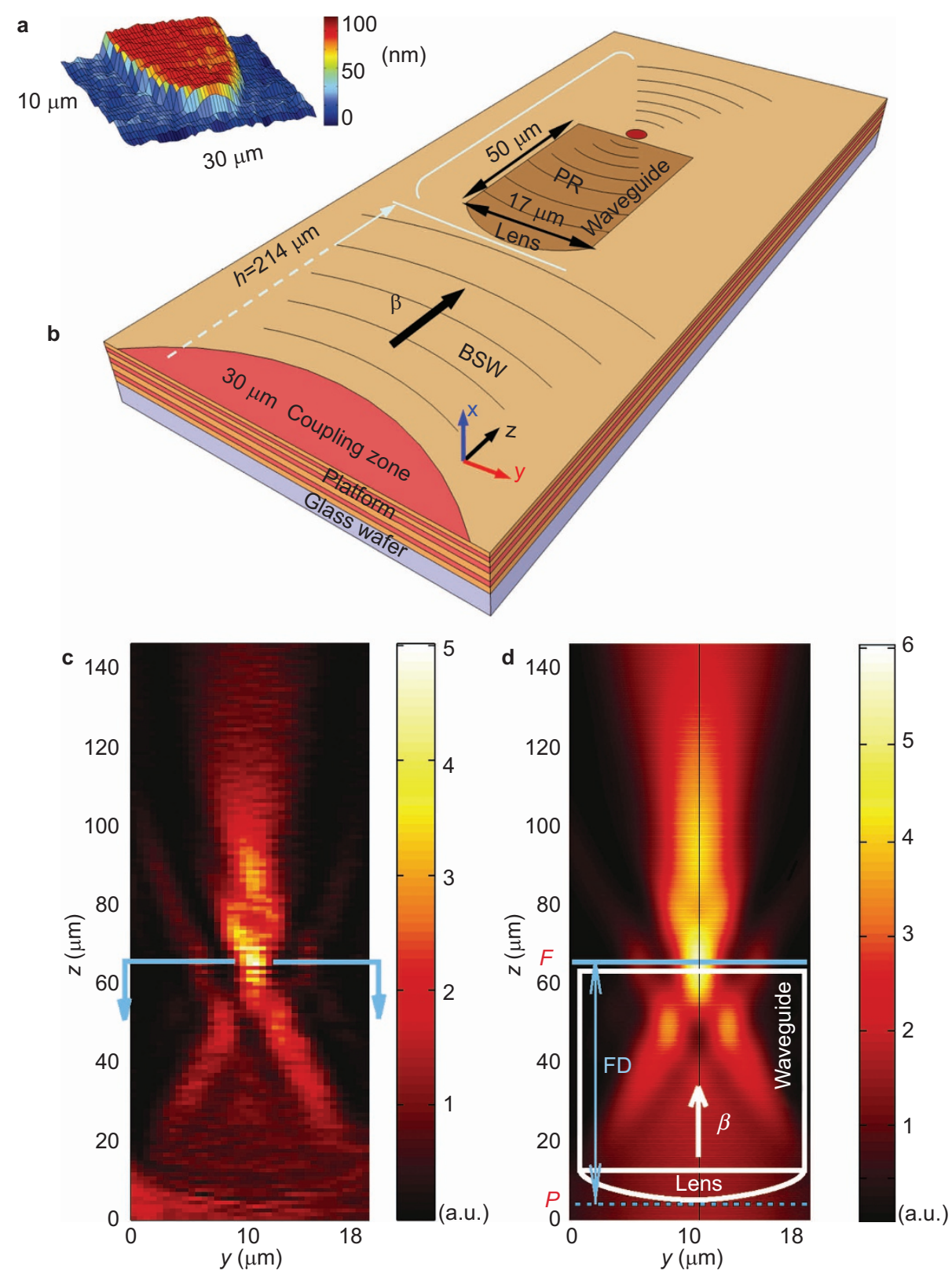

Figure 3 (a) Topography of the 100-nm-thick (layer-thickness) planoconvex lens. (b) Schematic of the 2D lens: the BSW propagating on the surface of the platform is focused by an ultrathin PR layer patterned as a planoconvex lens appended to a waveguide. (c) Measured and (d) simulated near-field intensity, showing light propagation through the 2D planoconvex lens. A focused spot is observed $60 \mu \mathrm{m}$ from the first interface of the lens, and the positions of the 2D planoconvex lens and the attached waveguide are marked by closed solid lines. Pindicates the first interface of the lens, $F$ indicates the $z$ position of the maximum intensity of the focal spot and $\beta$ is the propagation constant of the BSW. The cross-section line in (c) corresponds to the maximum measured intensity position. BSW, Bloch surface wave; 2D, two-dimensional; PR, photoresist.

For simplicity, we use the focal distance FD to the focal spot as a reference instead of the focal length $f$, which is similar according to our calculations. Lower Fresnel numbers indicate greater diffraction effects. In our case, the Fresnel number $N$ is equal to 0.258 and is much smaller than 1, which indicates that the behavior of this BSW-based 2D lens is dominated by diffraction. In our 2D lens model configuration, the refractive index contrast $\Delta n$ defines the order of the Fresnel number and, thus, the lens properties.

To compare the $2 \mathrm{D}$ lens with a $3 \mathrm{D}$ lens, we simplify our model to a thin planoconvex lens without an appended waveguide structure. Here, we define the focal shift as $\Delta f=\mathrm{FD}-f_{g}$. Assuming a plane wave incident beam, in Figure $4 \mathrm{a}$ and $4 \mathrm{~b}$, we simulate and theoretically compare the focal distance as well as the ratio $\Delta f / f_{g}$ for a $2 \mathrm{D}$ lens and a spherical $3 \mathrm{D}$ lens with the same depth and radius $R$ as a function of the refractive index contrast $\Delta n$. It can be seen that the focal distance and $\left|\Delta f / f_{g}\right|$ decrease as the refractive index contrast increases for both the $2 \mathrm{D}$ and $3 \mathrm{D}$ lenses. This result agrees with the study of Poon ${ }^{40}$ on the focal shift for $3 \mathrm{D}$ lenses. According to that study, the focal position for all $3 \mathrm{D}$ lenses with small $N$ shifts toward the lens, leading to a negative focal shift $(\Delta f<0)$. We can see the same behavior for a $2 \mathrm{D}$ lens, as presented in Figure 4b. Moreover, the ratio $\left|\Delta f l f_{g}\right|$ allows one to determine the squeeze of the focal spot towards the lens. The insets of Figure $4 \mathrm{a}$ and $4 \mathrm{~b}$ 

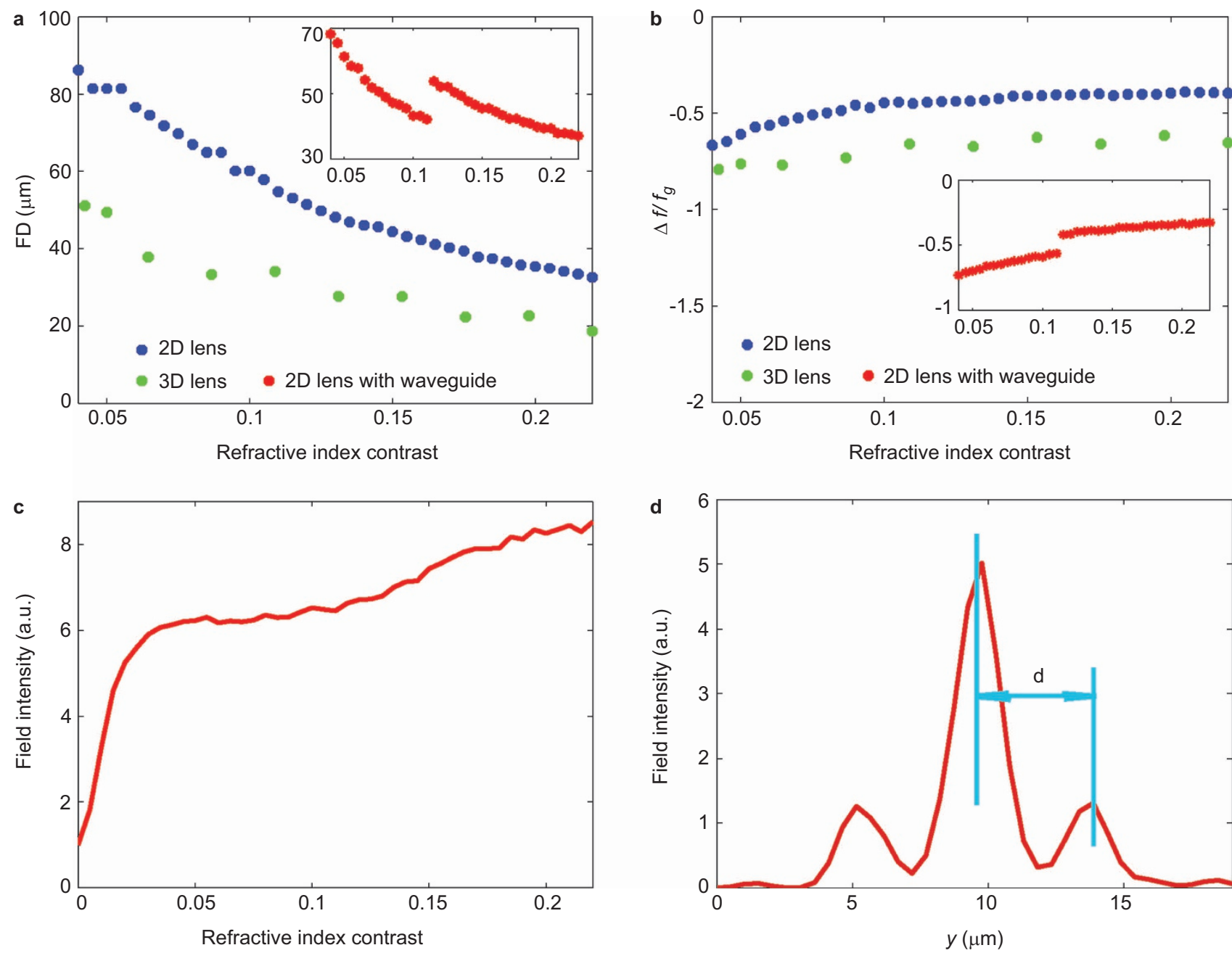

Figure 4 (a) FDTD simulation of the focal distance as a function of the refractive index contrast for the simplified 2D lens and the 3D lens model without a waveguide. The inset shows the focal distance of the 2D lens appended to a $50 \mu \mathrm{m}$-long waveguide. (b) Simulations of $\Delta f / f_{g}$ as a function of the refractive index contrast. (c) Simulated near-field intensity enhancement of the $2 \mathrm{D}$ lens appended to a $50 \mu \mathrm{m}$-long waveguide. The results are normalized by the incident beam at the first interface of the lens. (d) Cross-section of the measured intensity at the focal spot of the $2 \mathrm{D}$ lens appended to a $50 \mu \mathrm{m}$-long waveguide, corresponding to the line marked in Figure 3c. 2D, two-dimensional; 3D, three-dimensional; FDTD, finite-difference time-domain.

show the focal distance and the $\Delta f / f_{\mathrm{g}}$ ratio for the $2 \mathrm{D}$ lens appended to a $50-\mu \mathrm{m}$ waveguide, as described in Figure $3 \mathrm{~b}$. The disconnection appears after the total internal reflection condition is satisfied for a certain $\Delta n$ at the sidewall of the waveguide structure and is a result of interference.

The intrinsic difference between 2D and 3D lenses is displayed in Figure $4 \mathrm{~b}$. There is a difference of approximately $20 \%$ in the focal shift between the $2 \mathrm{D}$ and $3 \mathrm{D}$ lenses, which agrees with the aperture ratio between a concentric square and a circular area. The near-field intensity cross-section profile of the measured focal spot, as marked in Figure 3c, is plotted in Figure 4d. One can observe a Sinc function, confirming the $2 \mathrm{D}$ behavior of the lens. The resolution $d$ is defined as the distance of the peak-to-peak intensity in Figure 4d and can be deduced from

$$
d=\frac{\lambda}{2 n_{\mathrm{PR}}(R / \mathrm{FD})}
$$

Theoretically, we obtained a resolution of $d_{\text {calculate }}=4.38 \mu \mathrm{m}$, which is in good agreement with the measured value, $d_{\text {measured }}=4.36 \mu \mathrm{m}$. As shown in Equation (2), the resolution $d$ is proportional to the focal distance. Thus, the large focal shift gain increased the resolution.
In Figure 4c, we theoretically study the field confinement at the vicinity of the $2 \mathrm{D}$ lenses as a function of the refractive index contrast. The field confinement is defined as the ratio between the generated electric field normalized to the incident beam in front of the lens. The ratio increases as the refractive index contrast increases. Thus, the focusing capability of a 2D lens plays an important role in the surface wave field enhancement. At $\Delta n=0.055$, our experimental working point, the field intensity of the generated BSW is fivefold greater (Figure 3c), which indicates that the electric field at the PR layer is five times the intensity of the BSW generated without the lens. As described above, the intensity of the generated BSW is 100 times the intensity of the BSW generated for the TM case (Figure 1b). For our multilayer configuration, the near-field intensities for TE and TM polarizations can be directly compared. Therefore, we can deduce that the field intensity is enhanced by $5 \times 100$ at the focal spot position, compared with TM as a reference. This ability to localize a high field confinement on the surface compared with another polarization could be of special interest for sensing applications based on fluorescence or Raman spectroscopy. ${ }^{19,20,29}$ 


\section{CONCLUSIONS}

By manipulating BSWs with 2D photonic components, we demonstrate a BSW-supporting multilayer as a concept of a wafer-based dielectric platform for the first time, to our knowledge, where surface electromagnetic waves can be excited, directed and manipulated with $2 \mathrm{D}$ freedom. Photonic components, produced with a polymer on top of the platform, can be easily producible, accessible and controllable. The properties of the platform define the propagation of the surface modes and affect the manipulation capabilities of the surface components. To demonstrate the platform concept, a 2D lens on a BSW is verified as an example. The focal shift and resolution of the BSW 2D lens are measured. The near-field measurements are in agreement with theory, and a comparison with a $3 \mathrm{D}$ lens is performed.

Furthermore, we provide practical methods for increasing the effective refractive index contrast $\Delta n$, thus increasing the capability to control light on the platform. For the multilayer used in this work, one can optimize $\Delta n$ by either increasing the PR thickness up to $500 \mathrm{~nm}$ or by substituting the polymer with higher refractive index materials, such as metallic compounds (e.g., $\mathrm{TiO}_{2}$ with $n=2.2$ at $1550 \mathrm{~nm}$ ). In contrast, by carefully selecting the multilayer material and geometry, a $\Delta n$ of 0.5 can be achieved. These considerations will be addressed in forthcoming work. In additional, another possibility would be to include SPP structures in the platform, which may lead to significant opportunities for various applications.

\section{ACKNOWLEDGEMENTS}

This work was supported by the Swiss National Science Foundation. The authors would like to thank Emiliano Descrovi for his knowledge, his support in understanding the physical mechanism and calculations on the multilayer, and his revision of the manuscript. The authors would like to thank Toralf Scharf and Mme. Irène Philipoussis Fernandez for the photoresist deposition.

1 Knoll W. Interfaces and thin films as seen by bound electromagnetic waves. Annu Rev Phys Chem 1998; 49: 569-638.

2 Zayats AV, Smolyaninov II. Near-field photonics: surface plasmon polaritons and localized surface plasmons. J Opt A Pure Appl Opt 2003; 5: S16-S50.

3 Barnes WL, Dereux A, Ebbesen TW. Surface plasmon subwavelength optics. Nature 2003; 424: 824-830

4 Pincemin F, Maradudin AA, Boardman AD, Greffet JJ. Scattering of a surface plasmon polariton by a surface defect. Phys Rev B 1994; 50: 15261-15275.

5 Smolyaninov II, Mazzoni DL, Mait J, Davis CC. Experimental study of surface-plasmon scattering by individual surface defects. Phys Rev B 1997; 56: 1601-1611.

6 Sánchez-Gil JA. Surface defect scattering of surface plasmon polaritons: mirrors and light emitters. App/ Phys Lett 1998; 73: 3509-3511.

7 González MU, Weeber JC, Baudrion AL, Dereux A, Stepanov AL et al. Design, nearfield characterization, and modeling of $45^{\circ}$ surface-plasmon Bragg mirrors. Phys Rev $B$ 2006; 73: 155416

8 Li X, Tan Q, Bai B, Jin G. Experimental demonstration of tunable directional excitation of surface plasmon polaritons with a subwavelength metallic double slit. App/ Phys Lett 2011; 98: 251109

9 Liu Z, Steele JM, Srituravanich W, Pikus Y, Sun C et al. Focusing surface plasmons with a plasmonic lens. Nano Lett 2005; 5: 1726-1729.

10 Yin L, Vlasko-Vlasov VK, Pearson J, Hiller JM, Hua J et al. Subwavelength focusing and guiding of surface plasmons. Nano Lett 2005; 5: 1399-1402.

11 Devaux E, Laluet JY, Stein B, Genet C, Ebbesen T et al. Refractive micro-optical elements for surface plasmons: from classical to gradient index optics. Opt Express 2010; 18: 20610-20619.

12 Feng L, Tetz KA, Slutsky B, Lomakin V, Fainma Y. Fourier plasmonics: diffractive focusing of in-plane surface plasmon polariton waves. Appl Phys Lett 2007; 91: 081101.

13 Zentgraf T, Liu Y, Mikkelsen MH, Valentine J, Zhang X. Plasmonic Luneburg and Eaton lenses. Nat Nanotechnol 2011; 6: 151-155.
14 Bozhevolnyi SI, Volkov VS, Devaux E, Laluet JY, Ebbesen TW. Channel plasmon subwavelength waveguide components including interferometers and ring resonators. Nature 2006; 440: 508-511.

15 Wei $\mathrm{H}, \mathrm{Xu} \mathrm{H}$. Controlling surface plasmon interference in branched silver nanowire structures. Nanoscale 2012; 4: 7149-7154.

16 Yeh P, Yariv A, Hong CS. Electromagnetic propagation in periodic stratified media. I. General theory. J Opt Soc Am 1977; 67: 423-438.

17 Shinn M, Robertson WM. Surface plasmon-like sensor based on surface electromagnetic waves in a photonic band-gap material. Sens. Actuators B Chem 2005; 105: 360-364.

18 Sreekanth KV, Zeng S, Shang J, Yong KT, Yu T. Excitation of surface electromagnetic waves in a graphene-based Bragg grating. Sci Rep 2012; 2: 737.

19 Delfan A, Liscidini M, Sipe JE. Surface enhanced Raman scattering in the presence of multilayer dielectric structures. J Opt Soc Am B 2012; 29: 1863-1874.

20 Pirotta S, Xu XG, Delfan A, Mysore S, Maiti S et al. Surface-enhanced Raman scattering in purely dielectric structures via Bloch surface waves. J Phys Chem C 2013; 117: 6821-6825.

21 Yeh P, Yariv A, Cho AY. Optical surface waves in periodic layered media. Appl Phys Lett 1978; 32, 104-105.

22 Giorgis F, Descrovi E, Summonte C, Dominici L, Michelotti F. Experimental determination of the sensitivity of Bloch surface waves based sensors. Opt Express 2010; 18: 8087-8093.

23 Sinibaldi A, Danz N, Descrovi E, Munzert P, Schulz U et al. Direct comparison of the performance of Bloch surface wave and surface plasmon polariton sensors. Sens Actuators B Chem 2012; 174, 292-298.

24 Danz N, Sinibaldi A, Michelotti F, Descrovi E, Munzert P et al. Improving the sensitivity of optical biosensors by means of Bloch surface waves. Biomed Tech (Berl) 2012; 57: 584-587.

25 Liscidini M, Sipe JE. Analysis of Bloch-surface-wave assisted diffraction-based biosensors. J Opt Soc Am B 2009; 26: 279-289.

26 Paeder V, Musi V, Hvozdara L, Herminjard S, Herzig HP. Detection of protein aggregation with a Bloch surface wave based sensor. Sens Actuators B Chem 2011; 157: 260-264.

27 Konopsky VN, Karakouz T, Alieva E, Vicario C, Sekatskii SK et al. Photonic crystal biosensor based on optical surface waves. Sensors (Basel) 2013; 13: 2566-2578.

28 Michelotti F, Sciacca B, Dominici L, Quaglio M, Descrovi E et al. Fast optical vapour sensing by Bloch surface waves on porous silicon membranes. Phys Chem Chem Phys 2010; 12: 502-506.

29 Soboleva IV, Descrovi E, Summonte C, Fedyanin AA, Giorgis F. Fluorescence emission enhanced by surface electromagnetic waves on one-dimensional photonic crystals. Appl Phys Lett 2009; 94: 231122.

30 Ballarini M, Frascella F, Enrico E, Mandracci P, de Leo N et al. Bloch surface wavescontrolled fluorescence emission: coupling into nanometer-sized polymeric waveguides. Appl Phys Lett 2012; 100: 063305.

31 Ballarini M, Frascella F, de Leo N, Ricciardi S, Rivolo P et al. A polymer-based functional pattern on one-dimensional photonic crystals for photon sorting of fluorescence radiation. Opt Express 2012; 20: 6703-6711.

32 Yu L, Sfez T, Paeder V, Stenberg P, Nakagawa W et al. Concurrent polarization retrieval in multi-heterodyne scanning near-field optical microscopy: validation on silicon form-birefringent grating. Opt Express 2012; 20: 23088-23099.

33 Sfez T, Descrovi E, Yu L, Brunazzo D, Quaglio M et al. Bloch surface waves in ultrathin waveguides: near-field investigation of mode polarization and propagation. J Opt Soc Am B 2010; 27: 1617-1625.

34 Descrovi E, Sfez T, Dominici L, Nakagawa W, Michelotti F et al. Near-field imaging of Bloch surface waves on silicon nitride one-dimensional photonic crystals. Opt Express 2008; 16: 5453-5464.

35 Descrovi E, Sfez T, Quaglio M, Brunazzo D, Dominici L et al. Guided Bloch surface waves on ultrathin polymeric ridges. Nano Lett 2010; 10: 2087-2091.

36 Sfez T, Descrovi E, Dominici L, Nakagawa W, Michelotti F et al. Near-field analysis of surface electromagnetic waves in the bandgap region of a polymeric grating written on a one-dimensional photonic crystal. Appl Phys Lett 2008; 93: 061108.

37 Sfez T, Descrovi E, Yu L, Quaglio M, Dominici L et al. Two-dimensional optics on silicon nitride multilayer: refraction of Bloch surface waves. Appl Phys Lett 2010; 96: 151101.

38 Ulrich R. Theory of the prism-film coupler by plane-wave analysis. J Opt Soc Am 1970; 60: 1337-1350.

39 Lin J, Dellinger J, Genevet P, Cluzel B, de Fornel F et al. Cosine-Gauss plasmon beam: a localized long-range nondiffracting surface wave. Phys Rev Lett 2012; 109: 093904. 40 Poon TC. Focal shift in focused annular beams. Opt Commun 1988; 65: 401-406.

This work is licensed under a Creative Commons AttributionNonCommercial-ShareAlike 3.0 Unported license. To view a copy of this license, visit http://creativecommons.org/licenses/by-nc-sa/3.0 